\title{
Tendência secular em estatura: uma revisão da literatura
}

\author{
Secular height trend: a literature review
}

Gilberto Kac 1

1 Departamento de Nutrição Social e Aplicada, Instituto de Nutrição Josué de Castro, Universidade Federal do Rio de Janeiro. Av. Brigadeiro Trompowsky s/no, Bloco J, 2 o andar, Rio de Janeiro, RJ 21941-590, Brasil. gkac@gbl.com.br
Abstract This paper presents a literature review of secular height trends. The principal topics included were definition of the phenomenon, use of historical data sets on height as an alternative approach for monitoring economic changes, the main theoretical explanations of the phe nomenon, the beginning of military anthropometry, and past and current use in measuring secular height trends in recruits and children. The most important results showed that the positive secular trend can be attributed to environmental influences, especially improvements in heal th, economic, and social conditions. The mean reported rates in height increases varied with age, socioeconomic status, and country, so one must exercise caution in drawing inferences. World War II sl owed the trend in several countries, including Brazil, but the trend was stronger in the post-war period than in the beginning of the century. Finally, we observed that data from military anthropometry are the most common source for esti mating secular height trends.

Key words Secular Trend; Body Height; Anthropometry; Military Personnel; Nutrition

Resumo Esse trabal ho constitui-se em uma revi são de literatura sobre tendência secular em estatura (TSE). Os principais tópi cos abordados foram a definição do fenômeno, a utilização de dados históricos de estatura como estratégi a alternativa no monitoramento de mudanças econômicas, as princi pais explicações teóricas sugeri das para a ocorrência do fenômeno, o início da antropometria militar, sua utilização no passado e no presente, e a magnitude da tendência em recrutas e na população infantil. Os princi pais achados revelaram que a TSE tem sido atribuída, sobretudo, a influências ambientais, em particular, a mel horias nas condições sanitárias, econômicas e sociais. As taxas médi as de incremento variaram conforme a idade, o estrato sóci o-econômi co e o país considerado; portanto, deve-se ter cautel a nas comparações interpopulacionais. A Segunda Guerra Mundial reduziu a tendência em diversos países, entre el es o Brasil, mas as taxas de incremento no pós-guerra foram ai nda maiores do que as observadas no início do século. Constatou-se, por fim, que dados provenientes da antropometria militar são os mais utilizados para estimativas deTSE.

Palavras-chave Tendência Secular; Estatura; Antropometria; Militares; Nutrição 
Introdução

A presente revisão aborda a ocorrência de Tendência Secular em Estatura (TSE) com base na reduzida literatura produzida no Brasil, e, principalmente, enfocando a extensa produção acadêmica internacional. Em diversas situações, as investigações conduzidas pelo presente autor com recrutas da Marinha do Brasil constituíram-se em aporte teórico para algumas discussões.

Entre os tópicos discutidos, merece destaque a interessante abordagem proposta por um grupo de economistas liderados por Dr. Wiliam Fogel, da Escola de Economia de Chicago. Trata-se de uma estratégia metodológica que permitiu avaliar, com base em dados de estatura, a evolução das condições econômicas da população norte-americana dos séculos XVII eXVIII.

Outro tópico de destaque é a discussão sobre determinantes da TSE. Nesta seção, enfatiza-se principalmente o efeito das variáveis ecológicas. As explicações genéticas também são pontuadas, no entanto, dada sua pequena contribuição, bem como pela ausência de eco nos meios acadêmicos, esta foi mantida ao mínimo.

Por fim, apresenta-se uma extensa abordagem sobre a magnitude da TSE no Brasil e no mundo, assim como dados de investigações calcadas na Antropometria Militar. Vale frisar que não é objetivo deste texto comparar diretamente a presença de TSE em diversos países e contextos, o que certamente resultaria em grandes diferenças quanto ao início, direção, velocidade e extensão das mudanças. Esperase, no entanto, permitir que o leitor capture a dimensão com que a TSE ocorreu no mundo nos últimos 150 anos, bem como os diversos fatores que interferiram na sua ocorrência, como, por exemplo, a Segunda Guerra Mundial.

\section{Definição do fenômeno}

O fenômeno de Tendência Secular em Estatura (TSE) é definido como a ocorrência de alterações na idade em que se atinge uma determinada estatura na infância ou adolescência, ou a estatura final alcançada pela população adulta de um país (Van Wieringen, 1986). Este fenômeno pode ser positivo, negativo, ou, ainda, estar ausente (Malina, 1990). Exemplo da primeira situação é a observação comum de que crianças hoje são, em média, mais altas que algumas gerações anteriores, como observado por Monteiro et al. (1994) para o Brasil. Em contraste, verifica-se em alguns países em desenvolvimento que adultos e crianças possuem hoje estaturas mais baixas que gerações precedentes. Esteé o caso da população chilena, que experimentou redução em estatura média de 5,5 cm entre 1920 e 1960 (Malina, 1990). Tendência secular negativa em estatura também foi evidenciada para meninos guatemaltecos de alto estrato sócio-econômico entre 5 e 17 anos, medidos entre 1972 e 1990. A taxa de decréscimo foi de $0,48 \mathrm{~cm} /$ ano e correlacionouse estatisticamente com a redução no produto doméstico bruto total e com a diminuição na produção de alimentos (Bogin et al., 1996). Já a ausência de tendência secular pode decorrer de duas situações. Primeiro, o alcance por parte da população do seu potencial genético máximo de crescimento no que tange ao tamanho e tempo de maturação; segundo, condições ambientais insatisfatórias para permitir a ocorrência positiva de tendência secular (Malina, 1990). Nota-se, portanto, que o processo de tendência secular é estreitamente associado a variações das condições ambientais.

Tendência secular: estatura como parâmetro na avaliação das condições de vida

A investigação da tendência secular em estatura é considerada atualmente uma importante alternativa no monitoramento de mudanças nos padrões econômico e de saúde e nutrição, comparadas às obtidas com indicadores econômicos (Fogel et al., 1982; Tanner, 1982; Floud, 1984; Floud et al., 1990). Assim, a utilização de dados de estatura é uma estratégia metodológica que vem sendo explorada para caracterizar o padrão de vida das populações, tendo como embasamento os trabalhos de Villermé na França e Chadwick na Inglaterra no século XIX. Este pilar teórico fica bem caracterizado ao ser considerado o seguinte postulado de Villermé: "as circunstâncias queacompanham a pobreza atrasam a idade em que a estatura final é alcançada ediminuem a estatura adulta "(Villermé, 1829, apud Tanner, 1981:162).

A relação entre estatura e condições ambientais está bem documentada por uma série de estudos conduzidos por importantes pesquisadores, como Quetelet, Boas eTanner, entre outros. Segundo Eveleth \& Tanner (1990), medidas de estatura ou peso, assim como a estatura final atingida por uma população e as taxas de mudanças na estatura durante os anos de crescimento, "refl etem de forma acurada o estado da saúde pública de um país e o estado nutricional médio deseus cidadãos" (Eveleth \& Tanner, 1990:1). A relevância da estatura tam- 
bém foi considerada em uma recente publicação da Organização Mundial de Saúde: “em populações economi camente desprivi legi adas, a baixa estatura em adultos também poderia ser usada como um indicador da iniqui dade sócioeconômica" (WHO, 1995:72).

Um ponto relevante que deve ser destacado é a ampla disponibilidade de séries temporais com dados de estatura, principalmente na Europa e Estados Unidos, segundo uma série de categorias (ocupação, localização geográfica, estratos econômicos, escolaridade, entre outras). Esta característica permite o desenvolvimento de parâmetros mais refinados para avaliação de mudanças a longo prazo no padrão de vida, comparadas às obtidas com indicadores de renda per capita, além de adicionar, em mais de um século, análises sobre os efeitos exercidos por mudanças na economia na qualidade de vida das populações (Fogel et al., 1982; Fogel et al., 1983). A existência destas séries temporais deriva principalmente de registros militares, censos e mesmo inquéritos domiciliares. Constituem-se, portanto, uma estratégia alternativa para análises sobre o estado nutricional e qualidade de vida, complementando outros índices, como renda per capita e consumo de alimentos (Fogel et al., 1982; Fogel, 1986; Floud, 1984) ou como variável similar aos índices convencionais utilizados em estatísticas vitais (Van Wieringen, 1986).

\section{A relação entre mortalidade e a TSE}

Alguns estudos demonstraram que, mesmo com o declínio das taxas de mortalidade, diferenças no crescimento entre classes sociais persistem (Smith et al., 1980). Esta observação não chega a surpreender, visto ser o evento uma ocorrência comum a vários países. No Brasil, por exemplo, a mortalidade infantil vem declinando desde as décadas de 30/40 deste século e, no entanto, ainda permanecem elevados os diferenciais entre estratos de renda no que se refere a desnutrição por déficit estatural (Monteiro et al., 1995a).

O comportamento de queda na mortalidade infantil para as diferentes regiões do país nas últimas seis décadas (1930/86) é descrito por Simões \& Monteiro (1995). A década de 30 fica marcada como o ponto em que a queda da mortalidade infantil inicia-se no Brasil, ou, pelo menos, é o que se supõe, já que não estão disponíveis dados de períodos anteriores. $\mathrm{O}$ que se observou foi uma tendência geral de declínio para o país como um todo (71\%). No entanto, foram encontradas distintas taxas de re- dução segundo região, variando de $65 \%$ para o Nordeste a $79 \%$ para o Sul e Sudeste. Pode-se suspeitar que, nas décadas subseqüentes, meIhorias no estado nutricional também possam ser observadas, manifestadas, por exemplo, no aumento gradativo na estatura média da população infantil ou adulta.

De fato, dados de Kac (1998) para recrutas da Marinha do Brasil nascidos entre 1940 e 1965 demonstraram uma associação entre a queda na mortalidade infantil e aumento da estatura média adulta. Neste estudo, o autor reportou elevadas taxas de aumentos em estatura para o período 1940-1965, especialmente para o quinquênio 1960- 1965 (coeficiente angular =0,266; $p=0,045$ ). Ademais, segundo o teste de Sheffe, a única média de estatura que diferiu para todo o período foi a do último quinquênio.

Outros trabalhos também verificaram uma relação estreita entre o perfil de morbi-mortalidade e a ocorrência deTSE. Tanner (1966), em uma extensa revisão de mais de 100 trabal hos, constatou que o início da TSE em vários países coincidiu com o declínio nas taxas de mortalidade. Do ponto de vista quantitativo, a simulação realizada por Floud (1984) nos dá uma dimensão interessante. Segundo este autor, a redução de um óbito por mil nascidos vivos poderia significar um aumento de 0,02 cm na estatura média (Floud, 1984). Outra importante evidência provem de uma extensa série histórica para recrutas suecos, onde os autores verificaram que durante uma longa epidemia de cólera não ocorreram aumentos na estatura média (Sandberg \& Steckel, 1987).

A escassez de estudos diacrônicos sobre TSE no Brasil não se restringe a populações de recrutas, mas refere-se também a outros grupos populacionais. Desta forma, a hipótese em questão, qual seja, a existência de uma associação entre o início da queda na mortalidade e o subseqüente aumento na estatura, foi testada apenas com base em alguns poucos estudos. Isto porque são poucas as análises sobre TSE de recrutas ou mesmo de adultos ou crianças já realizadas, e as disponíveis tiveram sempre como alvo grupos particulares, como os casos examinados por Victora et al. (1989) e por Paiva (1994), ou referem-se a uma tendência contemporânea quando realizadas a nível nacional (Marcondes \& Marques, 1993) ou mesmo regional (Figueiró, 1994). Contudo, os resultados reportados por Kac (1998) apontaram para a validade desta hipótese, que, entretanto, merece ser melhor estudada com base em séries temporais para outros grupos populacionais e para a população em geral. 
Correntes explicativas e fatores determinantes da TSE

Nos últimos 150 anos, observou-se tendência progressiva para um aumento na estatura de indivíduos nos países industrializados ocidentais e em alguns países em desenvolvimento (Bakwin \& McLaughlin, 1964; Tanner, 1966; Roche, 1979; Van Wieringen, 1986; Eveleth \& Tanner, 1990; Malina, 1990; Monteiro et al., 1994). As taxas médias, sobretudo para populações européias, variam conforme a idade e o estrato sócio-econômico, alcançando em torno de 1,0 a $1,3 \mathrm{~cm} /$ década para crianças entre 5 e 10 anos; 1,9 a $2,5 \mathrm{~cm} /$ década durante a adolescência e 0,6 a 1,0 cm/ década no início da idade adulta (Tanner, 1966; Malina, 1990).

Os aumentos observados em estatura comumente têm sido atribuídos a melhorias nas condições sanitárias, econômicas e sociais. No entanto, influências genéticas como a heterose já foram postuladas, como veremos mais adiante. Para a maioria das populações, contudo, os dois fatores exógenos mais importantes que influenciam o crescimento são possivelmente a nutrição e a presença/ ausência de doenças.

De uma forma geral, as explicações teóricas oferecidas sugerem importantes contribuições do meio ambiente, desempenhando um forte papel o desenvolvimento econômico e social (Van Wieringen, 1986; Shatrugna \& Rao, 1987; Ji \& Ohsawa, 1993; Monteiro et al., 1994). As influências ambientais caracterizam-se, por exemplo, por um aumento na disponibilidade de alimentos, melhorias das condições sanitárias e cuidados em saúde, entre outros (Van Wieringen, 1986; Greiner \& Gordon, 1992).

Os estudos que têm como cerne a investigação de determinantes sócio-econômicos (classe social, tipo de trabalho, renda per capita, características da família) influenciando os padrões de crescimento e de TSE confirmam a importante contribuição dos mesmos. Steckel (1983), por exemplo, aponta a influência que a renda per capita exerce na estatura: o coeficiente de correlação entre estatura e o logaritmo de renda per capita foi de 0,84 , tendo como base estudos realizados em 16 países, sobretudo europeus e asiáticos. $O$ autor demonstrou também que um aumento de 0,1 no coeficiente de Gini pode reduzir a estatura em mais de $3,0 \mathrm{~cm}$ e que a diferença na estatura adulta média foi de quase $13,0 \mathrm{~cm}$ quando comparados indivíduos do sexo masculino com renda per capita de 150 e 5000 dólares. Sandberg \& Steckel (1987), por sua vez, investigaram a influência da história econômica na estatura de recrutas suecos entre 1760 e 1880 . Os autores con- cluíram que, à exceção de períodos de crise e outros percalços, como uma epidemia de cólera, o aumento da estatura média foi consistente com melhorias na história econômica em geral.

Outro importante determinante da TSE é a redução no tamanho da família, resultante de melhorias no nível sócio-econômico como um todo (Van Wieringen, 1986). Autores como Goldstein (1971) e Rona et al. (1978) evidenciaram que crianças provenientes de grandes famílias inglesas e escocesas apresentaram média de estatura inferior àquelas nascidas em pequenas famílias, mesmo após controle para variáveis sócio-econômicas. Estes mesmos autores observaram ainda uma associação entre o tipo de trabalho do pai (manual, não manual) e a estatura dos filhos, em favor dos mais qualificados. Um outro interessante estudo considerando estatura e classe social é o de Walker et al. (1988). Este autores analisaram uma amostra de 7735 homens de meia idade nascidos na Grã-Bretanha entre 1919 e 1939 e observaram diferenças em estatura de quase $6,0 \mathrm{~cm}$ entre as classes sociais extremas, assim como a presença de TSE para ambas as categorias de trabalhadores. No entanto, a diferença entre trabal hadores manuais e não manuais aumentou de 2,0 cm em 1920 para 3,0 cm em 1939. Já a estatura média de trabalhadores manuais nascidos em 1938 era a mesma que aquela de trabalhadores não manuais nascidos em 1920, demonstrando a existência de um diferencial de quase 20 anos na estatura entre estas categorias.

O estudo conduzido por Kac \& Santos (1997) em recrutas e alistados da Marinha do Brasil também revelou resultados que comprovam as diferenças em TSE conforme grau de escolaridade e regiões do pais. O coeficiente de regressão entre estatura e ano de nascimento foi positivo e estatisticamente significativo $(0,337$ $\mathrm{cm} /$ ano) para alistados com 8 a 11 anos de escolaridade, enquanto que para alistados com a mais baixa escolaridade ( 1 a 4 anos) a análise de regressão indicou um coeficiente negativo. As análises realizadas segundo regiões do pais são particularmente importantes no Brasil, dada a intensa heterogeneidade das mesmas. Desta forma, diferenças nas taxas de TSE entre regiões, como as observadas por Kac \& Santos (1997) e por Kac (1998), significam desigualdades sociais e econômicas.

Evidências da eliminação de diferenças na estatura entre classes sociais foram descritas apenas em países cujo desenvolvimento social é hoje de alto nível, como a Noruega, tornando a experiência deste país extremamente peculiar (Bruntland et al., 1980). Já na Grã Bretanha, 
por exemplo, apesar do elevado nível médio de vida, diferenças em estatura entre classes sociais ainda persistem (Whincup et al., 1988; Kuh et al., 1991).

Deve-se deixar claro que um conjunto bastante amplo e heterogêneo de variáveis ambientais explica em grande parte a ocorrência do fenômeno de tendência secular em estatura, no entanto, vejamos a teoria que postula influências genéticas na ocorrência deTSE. A explicação mais utilizada preconiza que aumentos nas combinações genéticas heterozigóticas na reprodução de animais e plantas levaria a um aumento no tamanho das espécies. É também conhecida como a teoria do vigor híbrido (Hulse, 1957; Hennenberg \& Van der Berg, 1990).

A indicação mais direta da ocorrência de heterose foi dada por Hulse (1957). Em seu estudo em vilarejos suíços, o autor constatou que filhos de pais provenientes de localidades distintas e exogâmicas, apresentaram estatura 2,0 $\mathrm{cm}$ superior do que aquelas crianças cujos pais eram oriundos de uma mesma localidade (vilarejos endogâmicos). Entre as principais limitações do estudo de Hulse, destaca-se o fato de que não estavam disponíveis medidas de estatura dos pais, de forma que não foi possível comprovar se as estaturas de filhos heterozigóticos diferiram mais do que a estatura de seus pais, em comparação com o outro grupo. Uma outra limitação foi a indicação do próprio autor de que existiam importantes diferenças culturais entre os grupos. Por fim, Hulse negou a existência de influências do tamanho da família na estatura, que era menor no grupo heterozigótico. Fica claro que a hipótese da heterose só poderia ser comprovada caso todos os fatores acima mencionados fossem excluídos.

Rejeitando a hipótese de heterose e de outras mudanças genéticas, como o presente autor prefere fazer, a ocorrência de TSE inevitavelmente recai sobre os diversos fatores ambientais discutidos no início desta seção.

\section{A magnitude da TSE}

A ocorrência de TSE positiva em estatura está bem documentada para países da Europa, além do Japão e Estados Unidos (Tanner, 1966; Takahasi, 1966; Greulich, 1976; Roche, 1979; Floud, 1984; Van Wieringen, 1986; Malina, 1990). É importante deixar claro, mais uma vez, que a descrição da magnitude da TSE nos diversos países deve ser cautelosamente interpretada, já que os contextos históricos, econômicos e sociais são distintos. Desta forma, as inferências devem ser mantidas no âmbito par- ticular de cada contexto, sobretudo temporal. Outro aspecto que também deve ser mencionado é a possibilidade de que estimativas de TSE baseadas em dados provenientes de populações militares apresentem viés de seleção. Essa discussão, no entanto, foge ao escopo desta revisão.

O ambiente criado principalmente nos anos seguintes à Segunda Guerra Mundial pode ser considerado como uma importante situação experimental para o estudo de efeitos adversos na ocorrência de TSE e no crescimento infantil. A escassez de alimentos, de serviços de saúde e de outros bens e serviços essenciais foram fatores responsáveis para que tendências seculares em estatura cessassem temporariamente em alguns países da Europa e no Japão (Takahashi, 1966; Valastovsky, 1966; Van Wieringen, 1986).

No Brasil, os efeitos da Segunda Guerra Mundial na ocorrência de TSE foram investigados por Kac (1998), com base em dados de estatura de recrutas da Marinha nascidos entre 1940 e 1965 . Este autor observou que, no decorrer da primeira metade da década de 40, houve um declínio na média de estatura de 1,1 $\mathrm{cm}$. Esta queda nas médias de estatura foram atribuídas aos efeitos da Segunda Guerra Mundial. O argumento reside no fato de que a influência exercida pelo ambiente na determinação da estatura adulta ocorre principalmente nos primeiros anos de vida (Waterlow, 1988; Tanner, 1989). Mesmo não estando envolvido di retamente na guerra, está claro que a conjuntura econômica foi desfavorável ao Brasil na época, como demonstraram dados de Oliveira \& Lobo (1992).

A investigação conduzida por Cernerud \& Lindgren (1991), baseando-se em registros escolares de estatura para quatro coortes nascidas em 1933, 1943, 1953 e 1963 em Estocolmo, demonstrou aumento estatural por década mais marcante para crianças nascidas entre 1933 e 1943, que para as coortes posteriores à Guerra. A diferença na estatura entre as coortes de 1933 e 1943 foi de 1,0 cm na idade de 7 e 10 anos e de quase $2,0 \mathrm{~cm}$ para crianças com 13 anos. A partir da coorte de 1943, crianças de 7 anos não mais apresentaram elevações na estatura, enquanto as com 10 e 13 anos reduziram os aumentos quase à metade. Um aspecto importante que deve ser considerado na avaliação de TSE nessas faixas etárias é o tempo de maturação sexual, que pode ter exercido influência nas diferenças observadas pelos autores.

Ocorreu uma rápida recuperação em relação a aumentos em estatura nos anos subseqüentes à Segunda Guerra Mundial, como de- 
monstram dados de escolares de Oslo (Brundtland et al., 1980). Estas crianças apresentaram um incremento estatural médio da ordem de $1,0 \mathrm{~cm}$ para meninos e de $1,4 \mathrm{~cm}$ para meninas entre 1960 e 1975. Tanner (1966) refere que após a guerra a TSE foi ainda maior do que a evidenciada na primeira metade do século XX em alguns países. Esta tendência também foi observada por Kac (1998) para dados contemporâneos (1970-77) da Marinha do Brasil.

Considerando-se em particular as populações asiáticas e seus descendentes migrantes, alguns estudos também apontaram como principais causas para a ocorrência de TSE as intensas mel horias nas condições sócio-econômicas (Greulich, 1976; Tanner et al., 1982).

Tanner et al. (1982) avaliaram a TSE, estatura sentada e comprimento das pernas em crianças japonesas entre 5 e 17 anos. Os resultados demonstraram que a estatura adulta aumentou 4,3 e 2,7 cm para homens e mulheres, enquanto que a estatura sentada praticamente não aumentou. Estes resultados, em conjunto com os de Udjus (Udjus, 1964, apud Tanner et al., 1982), demonstraram ser o incremento das pernas o principal responsável pela tendência secular nas medidas adultas de estatura.

No Brasil foram desenvolvidos até hoje dois inquéritos antropométricos de representatividade nacional. O primeiro foi conduzido em 1974/ 75 (Viacava et al., 1983), e o segundo foi realizado em 1989 (Inan, 1990). A análise sobre TSE realizada por Monteiro et al. (1994, 1995b) utilizou dados produzidos por estes inquéritos e teve como base três coortes, quais sejam, indivíduos nascidos entre 1951-53, 1966-68 e 1982-83. Estimou-se a estatura média aos 7 anos na segunda e terceira coorte e aos 22 anos na primeira e segunda coortes. Esta metodologia foi inicialmente proposta por Monteiro \& Torres (1993).

Os resultados para o país como um todo apontam aumentos superiores a 3,5 cm aos $6 \mathrm{e}$ 7 anos (comparação entre as coorte de 1967 e 1982). Tendências mais antigas (comparação das coortes de 1952 e 1967) revelaram um aumento para adultos jovens ( 22 anos) mais modesto, no entanto significativo, da ordem de 1,3 $\mathrm{cm}$ para homens e de $1,0 \mathrm{~cm}$ para as mulheres. Segundo os autores, os resultados encontrados para as crianças (2,4 cm/ década) são excepcionais, ultrapassando, por exemplo, os verificados para a Checoslováquia ( $1,0 \mathrm{~cm} /$ década) e China (1,8 cm/ década).

Análises sobre a evolução da estatura segundo as regiões do país apontaram para uma tendência sempre positiva. Novamente, as tendências mais recentes foram de maior magni- tude. O déficit médio em estatura aos 7 anos para meninas estudadas pela PNSN das regiões Sul, Sudeste e Centro-Oeste era de apenas 1,5 $\mathrm{cm}$ em relação às medianas do National Center for Health and Statistics, enquanto para as regiões Norte e Nordeste este valor atingia 5,0 a $7,0 \mathrm{~cm}$, respectivamente, em 1989. Quando a tendência foi avaliada segundo tercis de renda familiar per capita, observou-se claramente aumentos para todas estas categorias. Quando a análise foi realizada em relação a déficits do NCHS, apenas o tercil de maior renda aproximou-se da referência e, mesmo assim, ainda apresentou um déficit de 0,8 e $0,2 \mathrm{~cm}$ para meninos e meninas, respectivamente. Para o tercil de menor renda, este déficit foi da ordem de 6,0 a 7,0 $\mathrm{cm}$. Em suma, mesmo com a ocorrência de forte tendência secular em estatura, as crianças brasileiras de baixa renda não alcançam as medianas de estatura da referência norte-americana.

Koifman (1987) investigou a evolução da estatura de crianças entre 6 e 9 anos no bairro de Irajá, no Rio de Janeiro. Entre 1959 e 1970, o aumento foi de $2,6 \mathrm{~cm}$ aos 6 e 8 anos; $2,8 \mathrm{~cm}$ aos 7 anos e 1,0 cm aos 9 anos. Os aumentos em estatura foram marcados por uma descontinuidade para os nascidos em 1961-62, provavelmente devido a uma intensa recessão econômica neste período. Entre 1970 e 1971, verificou-se uma estabilidade nas médias de estatura, que, segundo o autor, pôde ser associada a um aumento na mortalidade infantil.

Percebe-se, portanto, que são escassos os estudos enfocando o processo de TSE no Brasil na população geral ou mesmo em populações particulares. Mesmo o estudo de Monteiro et al. (1994) só permitiu comparar a dinâmica do fenômeno a partir de dois recortes temporais. Por outro lado, é crescente o número de investigações que analisam o assunto com base em dados militares, como será discutido abaixo.

\section{Antropometria Militar}

Foram dois os motivos para que a aferição e o registro da estatura de recrutas passassem a ser rotina nas tropas européias e norte-americanas, onde se iniciou a antropometria militar. $\mathrm{O}$ primeiro deveu-se ao fato de que soldados mais altos eram geralmente mais fortes, cobriam maior espaço na marcha devido sua maior passada e, em combate, podiam alcançar mais Ionge com suas armas (Tanner, 1981). O segundo motivo relacionava-se à possibilidade de identificar um dado recruta a partir da medida de sua estatura em época na qual eram numerosos os desertores (Floud, 1984). 
O uso de dados antropométricos provenientes das Forças Armadas em pesquisas sobreTSE tem como um dos pilares os estudos de Villermé na França. Este autor sugeriu que mudanças na estatura média de uma nação eram sensíveis a condições nutricionais e ambientais, e permitiu que estas informações fossem utilizadas em análises sobre mudanças econômicas e sociais. Ainda no início do século XIX, Villermé analisou dados sobre a estatura de recrutas franceses e constatou que $45 \%$ do total de recrutas (1816-17) tinham estatura superior a $165 \mathrm{~cm}$, proporção esta que passou a 50\% no período 1826-27, levando-o a concluir que “o aumento na estatura entre 1816-1827 poderia derivar de outras causas, como um decréscimo, mesmo que pequeno, na miséria" (Villermé, 1829, apud Tanner, 1981:162).

Nos Estados Unidos foi a seleção de recrutas para a Revolução Americana (1775-1783) que estimulou o início da antropometria militar. A partir desta, variáveis como estatura, peso e o índice de massa corporal passaram a ser utilizadas na seleção de recrutas (Gordon \& Friedl, 1994).

A análise de dados antropométricos para a Revolução Americana apontou para uma estatura média de aproximadamente $173 \mathrm{~cm}$ (Sokoloff \& Villaflor, 1982). Esta média, referente a indivíduos entre 24 e 35 anos, leva a crer que, já nesta época, norte-americanos de cor branca estariam se aproximando dos patamares de estatura da era moderna. Esta afirmativa é corroborada pelo fato de que esta média era de 2,5 a 10,1 cm maior do que a reportada para diversos países europeus no final do século XVII e início do século XVIII, além de serem virtualmente idênticas às médias de estatura de recrutas alistados para a Segunda Guerra Mundial . Depreende-se deste fato que melhorias nutricionais ocorreram mais cedo e desenvolveramse de forma mais rápida nos Estados Unidos (Sokoloff \& Villaflor, 1982; Fogel et al., 1983).

A utilização crescente de grandes bancos antropométricos militares pode ser evidenciada pelo considerável número de trabalhos que se basearam nestes registros. Sua utilização hoje não mais se restringe à seleção de indivíduos para o serviço militar, sendo comum análises sobre TSE, como demonstraram diversos trabalhos (Terrenato \& Ulizzi, 1983; Floud, 1984; Victora et al. 1989; Sobral, 1990; Marcondes \& Marques, 1993; Figueiró, 1994; Paiva, 1994; Weber et al., 1995; Kac \& Santos, 1997; Kac, 1998).

Importantes análises de dados em estatura média de recrutas de países europeus no período de 1760 a 1975 podem ser encontradas em Floud (1984). O que se evidenciou para todos os países foi um considerável aumento na estatura média dos recrutas ao longo do tempo. Este aumento ocorreu, no entanto, de forma bastante diversa entre os vários países. Os recrutas holandeses foram os que apresentaram a maior estatura média no final do período (180,1 cm), seguidos dos recrutas suecos $(178,4$ $\mathrm{cm}$ ). Para estes países, a tendência foi, respectivamente, de 1,37 e 0,99 cm/ década. Em um bloco intermediário, com tendências de $0,88 \mathrm{~cm} /$ década, estão a Noruega e a França. As tendências para os demais países estudados foram as seguintes: Bélgi ca $(0,74 \mathrm{~cm} /$ década), Itália $(0,64$ $\mathrm{cm} /$ década) e Dinamarca $(0,53 \mathrm{~cm} /$ década).

Floud (1984) considerou ainda, por meio de análise de regressão, a relação entre estatura, taxa de mortalidade infantil e produto per capita doméstico bruto. A equação de regressão foi desenhada de forma que o grupo para comparações interpopulacionais fosse constituído pela experiência da população italiana. Os resultados demonstraram que, em conjunto com as demais variáveis da equação (variáveis “indicadoras" utilizadas para averiguar se a experiência para os demais países diferiu daquela da população italiana), a taxa de mortalidade infantil e o produto per capita doméstico bruto explicaram 96\% das diferenças na estatura entre as populações européias desde 1880. Ao se considerar estas variáveis separadamente (assumindo-se que as demais mantiveram-se constantes), os resultados demonstraram que o aumento de um dólar no produto per capita doméstico (constante para os preços de 1970) estaria acompanhado de um incremento na estatura da ordem de $0,003 \mathrm{~cm}$ (Floud, 1984).

A evolução da estatura de recrutas italianos no período entre 1874 e 1960 foi apresentada por Terrenato \& Ulizzi (1983). O incremento total foi de $9,0 \mathrm{~cm}$ ( 163,8 para $172,8 \mathrm{~cm}$ ) e refere-se a quase totalidade da população masculina italiana. Já em Portugal, a investigação realizada por Sobral (1990) observou maiores aumentos em estatura no litoral $(6,1 \mathrm{~cm})$ em comparação às regiões do interior (4,4 e 4,6 cm). Weber et al. (1995) analisaram a TSE para 700.000 homens austríacos que se alistaram entre 1980 e 1993. Os autores verificaram um aumento de $0,8 \mathrm{~cm}$ no período, o que significa uma taxa de 0,53 $\mathrm{cm} /$ década. Análises entre os estratos urbano/rural apontaram para uma diminuição na diferença de 1,2 para 0,4 cm, indicando que este diferencial tende a desaparecer no ano 2000.

No estudo de Victora et al. (1989) foi analisada a TSE de recrutas de três municípios do Sul do Brasil (Pelotas, Rio Grande e Bagé) entre 1940 e 1969. Os resultados apontaram para um aumento na estatura média de $4,0 \mathrm{~cm}$, com épo- 
cas de pequeno decréscimo (1943/ 47 e 1957/ 60) e de estabilização (1960-1967), decorrentes, segundo os autores, de dificuldades econômicas. As variações na estatura estiveram intimamente relacionadas com as tendências apresentadas pelo salário mínimo real $(r=0,63)$, o que explicou muitas das quedas e dos incrementos. Para o coeficiente de mortalidade infantil esta relação não ocorreu $(r=-0,54)$.

Marcondes \& Marques (1993) estudaram a evolução da estatura em indivíduos aptos para o serviço militar (Exército) entre 1979 e 1991 em 24 estados brasileiros. Os resultados demonstraram que ocorreu uma el evação na estatura média para a grande maioria dos estados. Os maiores incrementos para os indivíduos com o mais al to grau de escolaridade foram observados no Sergipe $(4,0 \mathrm{~cm})$, seguido de Minas Gerais (3,9 cm) e Mato Grosso (3,0 $\mathrm{cm}$ ). Não foram observados incrementos no Espírito Santo, Ceará, Amapá e Acre.

Os autores observaram ainda que nenhuma média de estatura, seja em 1979 ou em 1991, superou o percentil 50 da referência do National Center for Health Statistics (NCHS), que é de 178,6 cm. Quando a comparação foi feita em relação à referência Santo André, cuja média de estatura é de $173 \mathrm{~cm}$, sete estados (Minas Gerais, Bahia, Sergipe, Paraíba, Mato Grosso, Mato Grosso do Sul, Distrito Federal e Goiás) não superaram o percentil 50. Já em 1991, seis estados continuavam não superando o percentil 50 da referência Santo André (Paraíba, Ceará, Rio Grande do Norte, Piauí, Amazonas e Pará).

No estudo realizado por Paiva (1994) foi analisada a TSE de 4.447 indivíduos alistados na Junta Militar de Rio Branco, Acre, entre 1948 e 1974. A tendência foi positiva e o incremento médio em estatura foi de $0,07 \mathrm{~cm} /$ ano. $\mathrm{O}$ autor observou uma diminuição do déficit em relação ao NCHS de 14,5cm em 1962 para 9,6 em 1974 , no entanto, a maior média de estatura observada no período $(167,2 \mathrm{~cm})$ situava-se abaixo do percentil 10 do NCHS. Quanto ao local de nascimento, constatou-se uma tendência de crescimento semelhante entre os nascidos no Acre e em outros estados.

A relação entre estatura e salário mínimo foi inversa, enquanto o preço da borracha em dólar acompanhou a tendência crescente para estatura. A escolaridade aumentou $100 \%$ no período estudado, passando de 2,7 para 5,4 anos de estudo, e a relação entre escolaridade e estatura significou um aumento de $0,06 \mathrm{~cm}$ para cada ano a mais de escolaridade (Paiva, 1994).

Figueiró (1994) analisou a TSE em alistados no exército no estado de Pernambuco entre 1979 e 1990. A diferença na estatura entre o pri- meiro e o último ano estudado foi de $3,7 \mathrm{~cm}$ para o conjunto dos alistados, $3,6 \mathrm{~cm}$ para os residentes em áreas urbanas e $3,5 \mathrm{~cm}$ para os residentes em área rural. Foram observados aumentos em todas as categorias de escolaridade e de ocupação. As maiores variações em estatura foram encontradas para indivíduos com primeiro grau completo $(4,6 \mathrm{~cm})$. O maior incremento segundo ocupação foi observado entre trabalhadores não especializados $(6,2 \mathrm{~cm})$.

Segundo a autora, a tendência verificada entre os indivíduos alistados em Pernambuco entre 1979 e 1990 foi maior do que a média da região Nordeste, no entanto, inferior ao observado nas regiões mais desenvolvidas do país e nos países desenvolvidos. $O$ déficit de estatura para idade (<-2 escores $Z$ ) foi sempre superior ao observado para a população de referência norte-americana e variou de 45\% em 1979 a 24\% em 1990.

Kac \& Santos (1997) e Kac (1998), estudaram o fenômeno de TSE na população de recrutas e alistados da Marinha do Brasil entre 1940 e 1965 e durante a década de 70 (1970-77). Os resultados demonstraram importante aumento na estatura média para o Brasil como um todo no período 1940-65 (0,105 cm/ano), e valores substancialmente mais elevados para a tendência contemporânea $(0,315 \mathrm{~cm} /$ ano alistados e 0,266 - recrutas). Os autores concluíram, no entanto, que os resultados verificados para a Marinha devem ser vistos com cautela, dada a potencial presença de viés de seleção, e não podem ser diretamente extrapolados para a população brasileira, em face das diferenças existentes nas características antropométricas e sócio-econômicas de recrutas/ alistados e a população em geral.

Verificou-se, portanto, que ainda é escassa a literatura sobre TSE em populações militares do Brasil, quando comparada à literatura européia e norte-americana, o que, sem dúvida, demanda novas investigações sobre o tema.

\section{Conclusões}

Há fartas evidências na literatura enfatizando a relevância de análises de TSE como parâmetro/ procedimento para monitorar mudanças em saúde e nutrição. Os estudos de Fogel et al. (1982), Fogel (1986), Floud (1984), Floud et al. (1990) e Tanner (1982) demonstraram concretamente a potencialidade destes dados a partir de análises de grandes bancos de dados históricos.

São claras as inter-relações entre o perfil de morbi-mortalidade, o padrão de crescimento e 
a existência de TSE. Existe uma forte suspeita, ainda pouco testada no Brasil, de que a queda na mortalidade infantil coincidiria conseqüentemente com o início do processo de tendência secular em estatura. Segundo Tanner (1966), diversos estudos em países europeus também constataram esta relação.

Entre as correntes que tentam explicar o fenômeno, destaca-se a que sugere importantes contribuições do meio ambiente, desempenhando um forte papel o desenvolvimento econômico e social que pode ser traduzido, por exemplo, pelo aumento na disponibilidade de alimentos e maior acesso a serviços essenciais. As explicações genéticas não encontram respaldo nos meios científicos.

A revisão de literatura demonstrou ainda que a magnitude da TSE em crianças variou com a idade, país de nascimento, tipo de trabalho do pai e níveis de renda e que é escassa a literatura sobre o fenômeno no Brasil.

Existem fortes evidências, inclusive para o Brasil, de que a Segunda Guerra Mundial diminuiu a força daTSE. Por outro lado, após a guerra verificou-se em diversos países uma TSE positiva e ainda mais forte do que a observada na primeira metade do século XX.

Em relação a antropometria militar, as taxas reportadas também variaram entre os di- versos países e constatou-se que, mesmo com os problemas metodológicos existentes nestes bancos de dados, estas informações são as mais utilizadas na caracterização de TSE. Ou seja, dados de estatura provenientes de bancos militares são e sempre foram a forma mais comum de se estudar TSE em adultos.

Deve-se ter cautela em relação as comparações quanto a magnitude da TSE reportada pela literatura, já que as diversas investigações envolvem contextos dos mais variados. No entanto, quando o enfoque são populações militares, a comparação pode ser mais direta, observando sempre as relações temporais e os contextos sociais.

Ainda que inúmeros estudos sobre TSE já tenham sido realizados, uma série de questões ainda permanece em aberto, como, por exemplo, que mudanças nas condições ambientais do pós-guerra podem ser consideradas como determinantes da persistente presença de TSE naqueles países onde a tendência já era pronunciada? Será que existem diferenças sistemáticas na ocorrência de TSE entre os sexos? A interpretação da ocorrência de TSE é somente positiva ou existem aspectos negativos? Estas são perguntas importantes que somente estudos mais complexos serão capazes de responder.

\section{Referências}

BAKWIN, H. \& MCLAUGHLIN, S. M., 1964. Secular increase in height. Is the end in sight? Lancet, 1:1195-1196.

BOGIN, B.; KEEP, R. \& EDWARDS, R., 1996. Negative secular trend in stature for high SES Guatemalan boys. In: 21st Annual Meeting of the Human Biology Association, Abstracts. American Journal of Human Biology, 8:110-111.

BRUNDTLAND, G. H.; LIESTOL, K. \& WALLOE, L., 1980. Height, weight and menarcheal age of Oslo schoolchildren during the last 60 years. Annals of Human Biology, 7:307-322.

CERNERUD, L. \& LINDGREN, G. W., 1991. Secular changes in height and weight of Stockholm schoolchildren born in 1933, 1943, 1953 and 1963. Annals of Human Biology, 18:497-505.

EVELETH, P. B. \& TANNER, J. M., 1990. Worldwide Variation in Human Growth. Cambridge: Cambridge University Press.

FIGUEIRÓ, A. C., 1994. Perfil do Crescimento dos Jovens Alistados no Exército no Estado de Pernambuco entre 1979 e 1990. Dissertação de Mestrado, Recife: Departamento de Nutrição, Universidade Federal de Pernambuco. 
FLOUD, R., 1984. The heights of Europeans since 1750: A new source for European economic history. National Bureau of Economic Research, 1318: 1-31.

FLOUD, R.; WACHTER, K. \& GREGORY, A., 1990. Height, Health and History. Nutritional Status in the United Kingdom, 1750-1980. Cambridge: Cambridge University Press.

FOGEL, W. R.; ENGERMAN, S. L. \& TRUSSEL, J., 1982. Exploring the uses of data on height: The analysis of long-term trends in nutrition, labor welfare, and labor productivity. Social Science History, 6:401-421.

FOGEL, R. W.; ENGERMAN, S. L.; FLOUD, R.; FRIEDMAN, G.; MARGO, R. A.; SOKOLOFF, K. L.; STECKEL, R. H.; TRUSSEL, J.; VILLAFLOR, G. \&WATCHER, W., 1983. Secular changes in American and British stature and nutrition. Journal of Interdisciplinary History, 15:445-481.

FOGEL, R. W., 1986. Physical growth as a measure of the economic well-being of populations: the eighteenth and nineteenth centuries. In: Human Growth. A Comprehensi ve Treatise. Methodology, Ecological, Genetic and Nutritional Effects on Growth (F. Falkner \& J. M. Tanner, eds.), pp. 263281, New York: Plenum Press.

GOLDSTEIN, H., 1971. Factors influencing the height of seven years old children. Results from the national child development study. Human Biology, 43:92-111.

GORDON, C. C. \& FRIEDL, K. E., 1994. Anthropometry in the US armed forces. In: Anthropometry in the Individual and the Population (S. J. Ulijaszek \& C. G. N. Mascie-Taylor, eds.), pp. 179-210, Cambridge: Cambridge University Press.

GREINER, T. M. \& GORDON, C. C., 1992. Secular trends of 22 body dimensions in four racial/cultural groups of American males. American Journal of Human Biology, 4:235-246.

GREULICH, W. W., 1976. Some secular changes in the growth of American-born and native Japanese children. American Journal of Physical Anthropology, 45:553-568.

HENNENBERG, M. \& VAN DER BERG, E. R., 1990. Test of socioeconomic causation of secular trend: Stature changes among favored and opressed South Africans are parallel. American Journal of Physical Anthropology, 83:459-465.

HULSE, F. S., 1957. Exogamie et heterosis. Archives Suisses d'Anthropologie et Génétique, 22:103-25.

INAN (Instituto Nacional de Alimentação e Nutrição), 1990. Pesquisa Nacional sobre Saúde e Nutrição. Perfil de Crescimento da População Brasileira de 0 a 25 anos. Brasília: INAN.

JI, C. \& OHSAWA, S., 1993. Growth changes of Chinese Mongolian, Uygur, Koreans and Tibetan ethnics groups in the past forty years. American Journal of Human Biology, 5:311-322.

KAC, G. \& SANTOS, R. V., 1997. Secular trend in height in enlisted men and recruits from the Brazilian Navy born from 1970 to 1977. Cadernos de Saúde Pública, 13:479-488.

KAC, G., 1998. Tendência secular em estatura em recrutas da Marinha do Brasil nascidos entre 1940 e 1965. Cadernos de Saúde Pública, 14:109117.
KUH, D. L.; POWER, C. \& RODGERS, B., 1991. Secular trends in social class and sex differences in adult height. International Journal of Epidemiology, 20:1001-1009.

KOIFMAN, S., 1987. Crescimento em Escolares na Região delrajá, Rio de Janeiro. Décadas de 60 e 70. Tese de Doutorado, São Paulo: Faculdade de Medicina, Universidade de São Paulo.

MALINA, R. M., 1990. Research on secular trends in auxology. Anthropolgischer Anzeiger, 48:209227.

MARCONDES, E. \& MARQUES, R. M., 1993. Estudo antropométrico de indivíduos aptos para o serviço militar no período de 1979 a 1991. Revista Brasileira de Crescimento e Desenvolvimento Humano, 2:9-38.

MONTEIRO, C. A. \& TORRES, A., 1993. Can secular trend in child growth be estimated from a single cross sectional survey? British Medical Journal, 305: 797-799.

MONTEIRO, C. A.; BENÍCIO, M. H. D. \& GOUVEIA, N. C., 1994. Secular growth trends in Brazil over three decades. Annals of Human Biology, 2:381390.

MONTEIRO, C. A.; BENÍCIO, M. H. D.; IUNES, R. F.; GOUVEIA, N. C. \& CARDOSO, M. A. A., 1995a. Evolução da desnutrição infantil. In: Velhos e Novos Males da Saúdeno Brasil. A Evolução do Paíse de suas Doenças. (C. A. Monteiro, org.), pp. 93114, São Paulo: Editora Hucitec/ Núcleo de Pesquisas Epidemiológicas em Nutrição e Saúde, Universidade de São Paulo.

MONTEIRO, C. A.; BENÍCIO, M. H. D. \& GOUVEIA, N. C., 1995b. Evolução da altura dos brasileiros. In: Vel hos e Novos Males da Saúde no Brasil. A Evolução do País e de suas Doenças (C. A. Monteiro, org.), pp. 126-140, São Paulo: Editora Hucitec/ Núcleo de Pesquisas Epidemiológicas em Nutrição e Saúde, Universidade de São Paulo.

OLIVEIRA, A. \& LOBO, E. M. L., 1992. O Estado Novo e o sindicato corporativista, 1937-1945. In: Rio de Janei ro Operário: Natureza do Estado, a Conjuntura Econômica, Condições deVida e Consciência deClasse, 1930-1970 (E. M. L. Lobo, org.), pp. 102127, Rio de Janeiro: Access.

PAIVA, F. C. F., 1994. Estudo da Tendência de Crescimento dos Jovens Nascidos entre 1948 e 1974, Alistados no Serviço Militar em Rio Branco - Acre. Rio Branco: Departamento de Ciências da Natureza, Faculdade de Biologia, Universidade Federal do Acre. (mimeo.)

ROCHE, A. F., 1979. Secular trends in human growth, maturation, and development. Monographs of the Society for Research in Child Devel opment, 44:343.

RONA, R. J.; SWAN, A. V. \& ALTMAN, D. G., 1978. Social factors and height of primary schoolchildren in England and Scotland. Journal of Epidemiology and Community Health, 32:47-154.

SANDBERG, L. G. \& STECKEL, R. H., 1987. Heights and economic history. The Swedish case. Annals of Human Biology, 14:101-110.

SHATRUGNA, V. \& RAO, K. V., 1987. Secular trends in the heights of women from the poor community of Hyderabad. Annals of Human Biology, 14:375377. 
SIMÕES, C. C. \& MONTEIRO, C. A., 1995. Tendência secular e diferenciais regionais da mortalidade infantil no Brasil. In: Velhos e Novos Males da Saúdeno Brasil . A Evolução do país e suas doenças (C. A. Monteiro, org.), pp. 153-156, São Paulo: Editora Hucitec/ Núcleo de Pesquisas Epidemiológicas em Nutrição e Saúde, Universidade de São Paulo.

SMITH, A. M.; CHINN, S. \& RONA, R. J., 1980. Social factors and height gain of primary schoolchildren in England and Scotland. Annals of Human Biology, 7:115-124.

SOBRAL, F., 1990. Secular changes in southern Portugal between 1930 and 1980 according to conscript data. Human Biology, 62:491-504.

SOKOLOFF, K. L. \& VILLAFLOR, G. C., 1982. The early achievement of modern stature in America. Social Science History, 6:453-481.

STECKEL, R. H., 1983. Height and per capita income. Historical Methods, 16:1-7.

TAKAHASHI, E., 1966. Growth and environmental factors in Japan. Human Biology, 38:112-130.

TANNER, J. M., 1966. The secular trend towards earlier physical maturation. Tijdschrift Voor Sociale Geneeskunde, 44:524-538.

TANNER, J. M., 1981. A History of the Study of Human Growth. Cambridge: Cambridge University Press.

TANNER, J. M., 1982. The potencial of auxological data for monitoring economic and social well-being. Social ScienceHistory, 6:571-581.

TANNER, J. M., 1989. Fetus into Man. From Conception to Maturity. Cambridge: Harvard University Press.

TANNER, J. M.; HAYASHI, T.; PREECE, M. A. \& CAM ERON, N., 1982. Increase in length of leg relative to trunk in Japanese children and adults from 1957 to 1977: Comparison with British and with Japanese Americans. Annals of Human Biology, 9:411-423.

TERRENATO, G. \& ULIZZI, F., 1983. Genotype-environment relationships: An analysis of stature distribuition curves during the last century in Italy. Annals of Human Biology, 10:335-346.
VALASTOVSKY, V. G., 1966. The secular trend in the growth and development of children and young persons in the Soviet Union. Human Biology, 38: 219-230.

VAN WIERINGEN, J. C., 1986. Secular growth changes. In: Human Growth. A Comprehensive Treatise. Methodology, Ecological, Genetic and Nutritional Effects on Growth (F. Falkner \&J. M. Tanner, eds.), pp. 307-331, New York: Plenum Press.

VIACAVA, F.; FIGUEREDO, C. M. P. \& OLIVEIRA, W. A., 1983. A Desnutrição no Brasil: Uma Análise do Estudo de Despesa Familiar para o Nordeste, Estado deSão Paulo eEstado do Rio de Janeiro. Petrópolis: Editora Vozes.

VICTORA, C. G.; HORTA, L. B.; RAMOS, E. O. \& CARNIELETTO, G. E., 1989. Tendência secular ao crescimento em recrutas gaúchos, 1940-1969. Ciência e Cultura, 41:915-919.

WALKER, M.; SHAPER, A. G. \& WANNAMETHEE, G., 1988. Height and social class in middle-aged British men. Journal of Epidemiology and Community Health, 42:299-303.

WATERLOW, J. C., 1988. Observations on the natural history of stunting. In: Linear Growth Retardation in Less Devel oped Countries (J. C. Waterlow, ed.), pp. 1-16, Nestlé Nutrition Workshop Series No.14, New York: Raven Press.

WEBER, G.; SEIDLER, H.; WILFING, H. \& HAUSER, G., 1995. Secular change in height in Austria: An effect of population stratification? Annals of Human Biology, 22:277-288.

WHINCUP, P. H.; COOK, D. G. \& SHAPER, A. G., 1988. Social class and height. Lancet, 297:980-981.

WHO (World Health Organization), 1995. Physical Status: The Use and Interpretation of Anthropometry. WHO Technical Report Series 854. Geneva: WHO. 